\title{
Vordergründige Nähe
}

\author{
Zur Kommunikationskultur von Politik- und Medieneliten in Deutschland
}

\section{Barbara Pfetsch / Eva Mayerhöffer}

Im Mittelpunkt des Beitrags stebt eine Analyse derpolitischen Kommunikationskultur in Deutschland auf Basis einer quantitativen Befragung von 360 hochrangigen Politikern, politischen Sprechern und Journalisten. Wechselseitige Machtperzeptionen und Rollenwabrnebmungen dieser Akteure werden als „kognitive Geschäftsgrundlage" der Interaktion von Politik und Medien konzeptualisiert. Zentrale Frage ist, wie sebr die Orientierung an Medienlogiken ein durchgängiges Merkmal der politischen Kommunikationsbeziehungen ist und inwieweit Diskrepanzen in den Einstellungen medialer und politischer Akteure bestehen. Die empirischen Ergebnisse verweisen auf eine politische Kommunikationskultur, die sich nicht in einem einseitigen Postulat publizistischer Imperative auf der Seite medialer Akteure und einem analogen Postulat politischer Imperative auf der Seite der Politik manifestiert. Vielmehr werden medienorientierte Aufmerksamkeitsstrategien und Rollenkonzepte von beiden Seiten geteilt. Gespalten ist die Kommunikationskultur an jenen Stellen, an denen (macht)politische Rationalitäten ins Spiel kommen.

Schlagwörter: Politische Kommunikation, Kommunikationskultur, Medienmacht, Interaktion, Berufsnormen, Rollenverständnis

\section{Einleitung}

Mediensysteme unterscheiden sich nicht nur durch das Angebot an Medien und Medieninhalten, sondern insbesondere auch durch kulturell geprägte Stile der Politikvermittlung. Als Erklärungsmuster für die länderspezifische Variation der politischen Kommunikationspraxis verweist die Kommunikatorforschung auf unterschiedliche Journalismuskulturen (Hanitzsch 2007). Auf der anderen Seite argumentieren Hallin und Mancini (2004) überzeugend, dass die mediale Darstellung von Politik nicht nur durch die Organisation und das Verhalten der Medien, sondern genauso durch das Kommunikationsverhalten der politischen Eliten geprägt wird. Die jeweils spezifische politische Kommunikationspraxis eines Landes hängt also nicht zuletzt davon ab, wie sich die Beziehungen zwischen Journalisten und politischen Eliten gestalten, wie diese Gruppen ihre Rollen definieren und welche Regeln sie sich bei der Darstellung und Vermittlung politischer Vorgänge geben. Diese Überlegung ist nicht nur für den internationalen Vergleich von politischen Kommunikationskulturen wichtig, sie hilft auch, die politische Kommunikationspraxis im eigenen Land zu verstehen.

Die Einsicht, dass Prozesse und Inhalte politischer Kommunikation immer auch vom Beziehungsgeflecht zwischen Macht- und Medieneliten geprägt sind, lenkt den Blick auf die systematische Analyse der kommunikativen Orientierungen dieser Gruppen. Erste Untersuchungen dazu, die Mitte der 1990er Jahre noch das Milieu der „Bonner Käseglocke“ abbilden, zeigen, dass das politisch-mediale Nahverhältnis in Deutschland durch eine (partei)politische Interaktionskultur geprägt war, in der die politische Logik die Richtschnur vorgab (Pfetsch 2003b). Schon damals deuteten sich aber Wandlungstendenzen an, da in den Orientierungen von Journalisten trotz eines mehr oder weniger konsensuellen Verhältnisses die divergenten professionellen Zielsetzungen immer wie- 
der zum Vorschein kamen. Während sich die politischen Sprecher in ihren Handlungsorientierungen im Bereich der Politikvermittlung nach wie vor an parteipolitische Machtkalküle hielten, begannen die Journalisten, ihre publizistischen Ziele zur Richtschnur zu erheben. Die Diagnose war damals die Entwicklung einer „gespaltenen politischen Kommunikationskultur", die von der Spannung zwischen politischer Logik und publizistischen Motiven geprägt war (Pfetsch 2003b) ${ }^{1}$.

Seit den 1990er Jahren haben sich in Deutschland Regierungskonstellationen, Parteien und auch die Medienlandschaft wesentlich verändert. Dies hat nicht nur einen anhaltenden, vielfältigen Diskurs über die Mediatisierung der Politik provoziert (vgl. dazu Pfetsch \& Marcinkowski 2009), sondern auch - so Kepplinger (2009a: 52) - zu einer Kommunikationskultur geführt, die auf dem Weg hin zur Unterwerfung der Politik unter die Erfolgsbedingungen der Medien sei. In dieser Sicht wächst der Spielraum für eine Mediatisierung der Politik mit den Unterschieden in den Orientierungen von Politikern und Journalisten (Kepplinger 2009a: 44). Wenn diese Einschätzung zutrifft, dann steigt mit dem Ausmaß der Diskrepanzen im Milieu der politischen Kommunikation die Mediatisierung von Politik, was zu einer „Schmälerung der Grundlagen genuin politischer Macht“ (Kepplinger 2009a: 46) führt. Dieses Szenario mag für publizistische und politische Beobachter als eine ausgemachte Sache erscheinen, überzeugende empirische Studien über den Umfang und die Dimensionen der Distanzen liegen indessen kaum vor. Alternativ kann argumentiert werden, dass sich die Mediatisierung der Politik auch an übereinstimmenden Orientierungen von Politikern und Journalisten festmachen lässt. In dieser Sichtweise steigt der Mediatisierungsspielraum, wenn sich die Handlungsorientierungen der Politiker bezüglich der Kommunikation politischer Botschaften an journalistische Wahrnehmungen angleichen.

Vor diesem Hintergrund ist das Ziel unserer Studie, die gegenwärtige Kultur der politischen Kommunikation, die das Verhältnis von medialen und politischen Akteuren trägt, empirisch zu untersuchen ${ }^{2}$. Empirische Indikatoren sind die Perzeption politischer Medienmacht sowie professionelle Rollenzuschreibungen, die wir als die „kognitiven Geschäftsgrundlagen“ der Interaktion von Politikern und Journalisten in Berlin beschreiben. In unserer empirischen Untersuchung fragen wir:

- Wie nehmen Politiker, Journalisten und politische Sprecher die Rahmenbedingungen und Zielsetzungen der politischen Kommunikation wahr, wie schätzen sie die Medien als politische Einflussgröße auf ihre Rolle und ihr Handeln ein?

- Wie sehen sie sich selbst und wie die Rolle ihrer jeweiligen Gegenüber im alltäglichen Umgang?

- Und schließlich: Welche Spannungen und Konfliktpotenziale ergeben sich aus möglicherweise widersprüchlichen Rollen und Orientierungen?

Um die Einstellungsmuster der Akteure theoretisch zu fundieren und empirisch zu erfassen, arbeiten wir mit dem Konzept der Politischen Kommunikationskultur (Pfetsch

1 Der Begriff „gespaltene Kommunikationskultur“ bezieht sich dabei auf den Gegensatz zwischen politischer Logik, bezugnehmend auf die Rationalitäten politischer Auseinandersetzung, und medialer Logik, welche sich an Aufmerksamkeitskriterien und Nachrichtenwerten orientiert (vgl. dazu Altheide \& Snow 1979, Mazzoleni 1987), nicht etwa auf eine strukturell vorgegebene Spaltung zwischen politischen und medialen Akteuren. Es ist vielmehr eine empirische Frage, welche der Logiken für die Akteure politischer Kommunikation jeweils prägend ist.

2 Vgl. zur politischen Kommunikationskultur in Österreich die Untersuchung von Plasser und Lengauer (2010) sowie für die Schweiz die Untersuchung von Schwab Cammarano et al. (2010), zum internationalen Vergleich vgl. Pfetsch et al. (2009), Mayerhöffer (2009), Esmark \& Mayerhöffer (2010) und Maurer (2011). 
2003b, 2008). Danach wird die Interaktion von Politik und Medien auf der Grundlage von gemeinsamen Normen und Koorientierungen in Bezug auf die Kommunikationsziele und die Publika politischer Botschaften reguliert (Blumler \& Gurevitch 1981, 1995 : 240ff.). Für die empirische Forschung ergeben sich aus dieser Perspektive zwei Anforderungen:Zum einen der Akteursbezug, d. h. die politische Kommunikationskultur wird anhand der Einstellungen der sie tragenden Akteure - Journalisten, politische Sprecher und Politiker im Zentrum des Regierungssystems - erfasst. Unsere Studie geht hier über bisher vorgelegte Untersuchungen hinaus, indem sie nicht nur Journalisten (Donsbach \& Patterson 2003) bzw. Journalisten und Politiker (Kepplinger 2009b) einbezieht, sondern darüber hinaus politische Sprecher als dritte Gruppe systematisch berücksichtigt (vgl. auch Maurer \& Mayerhöffer 2009). Diese „Politikvermittlungsexperten“ (Tenscher 2003) erfüllen eine wichtige Brückenfunktion zwischen Journalisten und Politiker, weshalb es für eine umfassende Diagnose des politischen Kommunikationsmilieus notwendig ist, auch ihre Einstellungsprofile zu erfassen. Offen ist hier die Frage, ob sich in den Orientierungen und Rollenbildern von Sprechern eher die ideologischen Positionen der Politiker abbilden oder die kommunikativen Zielsetzungen der Medien. Zum anderen ist die politische Kommunikationskultur - im Gegensatz zur politischen Kultur der Bevölkerung - durch einen Elitebezug gekennzeichnet, d. h. wir erfassen die Grundlagen der politischen Kommunikation, indem wir auf die Einstellungen von Akteuren im Zentrum politischer und publizistischer Macht fokussieren.

Unser Beitrag ist in zwei Teile gegliedert. Zunächst diskutieren wir den Forschungsstand und erläutern unseren theoretischen Ansatz sowie die konkreten empirischen Fragestellungen. Als wesentliche Dimensionen politischer Kommunikationskultur betrachten wir die medialen Einflussüberzeugungen und ihren Zusammenhang mit Öffentlichkeitsstrategien, Rollenüberzeugungen und Konfliktmustern. Unsere Hypothese ist, dass die gegenwärtige politische Kommunikationskultur der Politik- und Medieneliten in Berlin sich nach wie vor durch die unterschiedlichen Imperative von Publizistik und Politik charakterisieren lässt. Entgegen der Vermutung Kepplingers erscheint es uns aber fraglich, ob die Mediatisierung ein durchgängiges selbst evidentes Strukturmerkmal der kognitiven Geschäftsgrundlagen von Medien- und Machteliten ist. Wir vermuten vielmehr, dass politische Logiken auch weiterhin Prägekraft für die politische Kommunikationskultur in Deutschland haben, vor allem für die Einstellungsmuster politischer Entscheidungsträger. Eine offene Frage ist aber, inwieweit dies auch für die politischen Sprecher gilt, die auf professionelle Weise mit den Kommunikationsansprüchen der Medien an die Politik konfrontiert sind. Möglicherweise sind es gerade die Sprecher, die durch eine Abkehr von der politischen Logik die Mediatisierung von Politik befördern.

Die empirische Grundlage unserer Studie ist eine Befragung von 360 Politikern, politischen Sprechern und Journalisten in Spitzenpositionen von Politik und Hauptstadtmedien aus dem Jahr 2008/2009. Die Daten stammen aus der deutschen Teilstudie eines international vergleichenden Projektes ${ }^{3}$, welches die politischen Kommunikationskulturen in neun europäischen Demokratien empirisch zu bestimmen und zu typologisieren versucht.

3 Das Projekt „Political Communication Cultures in Western Europe - A Comparative Perspective" wird im Rahmen des ECRP II Programms der European Science Foundation gefördert (www.communication-cultures.eu) und für Deutschland von der Deutschen Forschungsgemeinschaft finanziert. Die deutsche und international vergleichende Studie wird von Barbara Pfetsch (Freie Universität Berlin) koordiniert. 


\section{Theoretische Überlegungen}

\subsection{Forschungsstand}

Die Forschung in Deutschland über das Zusammenspiel von Medien und Politik hat sich über lange Zeit auf eine vorwiegend theoretische Diskussion beschränkt. Das Ziel waren Modelle zur Beschreibung der Machtverhältnisse, der Distanz und Nähe, der Autonomie und der Interaktion. Je nach Diktion wurde hier mit Dependenz-, Instrumentalisierungs- und Determinationstheorien oder in systemtheoretischer Perspektive mit Ansätzen der Interdependenz, Intereffikation und Interpenetration gearbeitet (vgl. zum Forschungsüberblick Baugut \& Grundler 2009: 103-141, Jarren et al. 1996: 20-23, Schulz 2008). Obwohl der akademische Diskurs zu einer Ausdifferenzierung der Theorien führte, verstellte er lange den systematischen Blick auf den empirischen Gegenstand, nämlich die Interaktion selbst sowie ihre kognitiven Voraussetzungen und Folgen. Allenfalls wurde das Zusammenspiel von Journalismus und Politik bei Skandalisierungen (Donsbach et al. 1993) oder einzelnen Politikbereichen (Harmgarth 1997, Rinke et al. 2006) untersucht. Oft blieben Studien harmlose Beobachtungen eines Mikrokosmos, die aufgrund sehr geringer Fallzahlen oder problematischer Stichproben kaum Anspruch auf Repräsentativität erheben können (z. B. Baugut \& Grundler 2009). Die ersten systematischen Bestandsaufnahmen politischer Kommunikationsmilieus konzentrierten sich einseitig auf die Seite der Journalisten und betrachteten Politik lediglich als ein Objekt journalistischer Einstellungsmuster und Handlungsweisen (Donsbach \& Patterson 2003, Weischenberg et al. 2006). Arbeiten über politische Sprecher sind ebenso einseitig auf deren Welt- und Berufswahrnehmungen bezogen (Tenscher 2003). Empirische Studien, die beide Seiten systematisch betrachten, sind mit Ausnahme der Studien von Kepplinger (2009a, 2009b) selten geblieben. Dagegen gibt es eine Vielzahl von Publikationen, die das Thema anekdotisch beleuchten (Bruns 2007, Hachmeister 2007, Rolke \& Wolff 2003). Eine erste empirische Bestandsaufnahme der das beiderseitige Verhältnis prägenden Einstellungen liegt mit den Arbeiten von Pfetsch (2003a, 2003b) vor. Im Vergleich mit den USA zeigte sich für die politische Kommunikationskultur Mitte der 90er Jahre, dass in Deutschland eine eher parteipolitisch geprägte Form politischer Kommunikationskultur vorherrschend war. Diese war von den Regeln der Politik geprägt sowie von sozialer Nähe zwischen politischen Sprechern und Journalisten. Schon damals war aber erkennbar, dass sich diese Geschäftsgrundlage für Deutschland aufzulösen begann.

Theoretisch betrachtet können sich Diskrepanzen in der politischen Kommunikationskultur ergeben entweder

(a) aufgrund von unterschiedlichen professionellen Rollenauffassungen und Handlungszielen von Politikern, Sprechern und Journalisten;

(b) aufgrund von unterschiedlichen parteipolitischen oder ideologischen Orientierungen; oder

(c) dadurch, dass die Akteure auf der Vorderbühne und auf der Hinterbühne unterschiedlich agieren (Hoffmann 2003, Lesmeister 2008).

Darüber hinaus ist zu beachten, um welche Einstellungen bzw. Einstellungsobjekte es sich handelt, $d$. h. ob es sich um allgemeine Einschätzungen der Machtverteilung zwischen Politik und Medien handelt oder um spezifische Orientierungen hinsichtlich der Berufsrollen und Normen der politischen Kommunikation.

In der Tat stützen unterschiedliche Arbeiten den Befund von divergierenden Einstellungsmustern zwischen Journalisten und Politikern, die entweder professionell, politisch-ideologisch oder sozial begründet sind. Hoffmann (2003), der die „Interpenetrationszone" zwischen Eliten aus Politik und Journalismus untersucht, zeigt, dass diese 
sich ihrer wechselseitigen Abhängigkeit sehr bewusst sind und dies auch auf der „Vorderbühne" offen thematisieren. Politiker fühlen sich dabei stärker abhängig von Journalisten als umgekehrt. Im Gegensatz zu dieser Wahrnehmung räumen jedoch beide Seiten den Politikern das größere Themensetzungspotenzial ein. Auf Diskrepanzen in der allgemeinen Wahrnehmung beider Gruppen verweist auch Kepplinger (2009b). In seiner Studie sind Journalisten und Politiker gleichermaßen der Meinung, dass die Medien mehr Einfluss auf die Politik besitzen als umgekehrt. Er interpretiert dies als Beleg einer Entwicklung in Richtung einer mediengetriebenen politischen Kommunikationskultur in Berlin.

In Bezug auf die gegenseitige Wahrnehmung eigener und fremder Rollen bzw. Handlungsorientierungen zeigt sich bei Hoffmann (2003) eine erhebliche Diskrepanz zwischen (positiv besetztem) Selbst- und (negativ besetztem) Fremdbild. Bei dieser Studie sind die negativen Einstellungen von Politikern gegenüber Journalisten stärker ausgeprägt als umgekehrt. Bei Kepplinger (2009b) indessen weisen die Journalisten das negativer geprägte Bild auf die Gegenseite auf. Die Abgrenzung des eigenen Rollenbilds manifestiert sich jedoch nicht nur in Bezug auf die jeweils andere Berufsgruppe, sondern auch in Abgrenzung zu Akteuren und Organisationen des eigenen Sektors. Rollenkonflikte entstehen vor allem dort, wo soziale oder ideologische Erwartungen auf der einen und professionelle Erwartungen auf der anderen Seite aufeinandertreffen.

Auf Diskrepanzen, die insbesondere in der „informellen politischen Kommunikationskultur" begründet sind, verweist indessen Lesmeister (2008). Sie zeigt, dass sich die Wahrnehmungen des Machtverhältnisses zwischen Politik und Medien sowie auch professionelle Rollenbilder gänzlich anders gestalten, wenn der Bezugspunkt von der „Vorder-“ auf die „Hinterbühne“ politischer Kommunikation wechselt. So verliert die Frage nach einem Machtverhältnis und gegenseitigen Einflusspotenzialen in der informellen Kommunikation ihre Relevanz; sie wird vielmehr durch eine Denkweise in Kategorien des gegenseitigen Nutzens ersetzt. Auch das Denken in Berufsrollen tritt im informellen Rahmen in den Hintergrund: Mit Ausnahme der Selbstwahrnehmung der journalistischen Akteure lassen sich kaum generalisierbare Rollenbilder identifizieren, das Gegenüber wird vielmehr als Individuum außerhalb seiner Berufsrolle wahrgenommen.

\subsection{Dimensionen der politischen Kommunikationskultur}

Aus den vorliegenden Studien sind zwei Schlussfolgerungen zu ziehen. Zum einen erweist sich in Bezug auf die empirische Erhebung der politischen Kommunikationskultur eine Dimensionierung als sinnvoll, welche die Einstellungsmuster der Akteure anhand von zwei Dimensionen strukturiert (Pfetsch 2003a): Dies ist zum einen die von Mazzoleni (1987) vorgeschlagene Unterscheidung zwischen der Ausrichtung der politischen Kommunikation nach der Medienlogik versus der politischen Logik. Diese manifestiert sich in Orientierungen über die Stärke bzw. Schwäche der Massenmedien in Bezug auf ihre politischen Funktionen. Es geht also um die Frage, wie die Rolle der Massenmedien im politischen Prozess eingeschätzt wird und wie dies die jeweiligen Öffentlichkeitsstrategien beeinflusst.

Die zweite Dimension bezieht sich auf die Wahrnehmung von Distanzen in den professionellen Rollen, d. h. politische Kommunikationskultur manifestiert sich in der Selbst-und Fremdeinschätzung der Akteure. Da die politische Kommunikationskultur ein grundsätzlich relationales Konzept ist, geht es hier jedoch nicht - wie bei reinen Kommunikator- oder politischen Elitestudien - allein um die (Selbst)wahrnehmung der eigenen Rolle. Vielmehr sollen die reziproken Sichtweisen auf die eigene Rolle um die Sicht auf die Rolle des Gegenübers sowie um die Wahrnehmung der Konflikthaltigkeit 
des gegenseitigen Verhältnisses ergänzt werden. Die Frage nach der Konstellation der Rollenwahrnehmungen zueinander erscheint als entscheidender Parameter einer politischen Kommunikationskultur, die aus normativer demokratietheoretischer Sicht für das Funktionieren politischer Öffentlichkeit und schließlich des politischen Prozesses nötig ist.

Zum anderen ergeben die vorliegenden Befunde unterschiedlicher Studien ein uneinheitliches Bild in Bezug auf die wesentlichen Charakteristika der politischen Kommunikationskultur in Deutschland. Wir wissen, dass sich Politiker und Journalisten gegenseitig in Haftung nehmen, wenn es um die Machtverhältnisse in der politischen Kommunikation geht. Allerdings ist nicht eindeutig, wer wen für einflussreicher und mächtiger hält und welche Konsequenzen dies hat. Wir wissen auch, dass die verschiedenen Studien in Bezug auf die Rollenerwartungen zu uneinheitlichen Befunden kommen. Insgesamt erscheint gegenwärtig nicht ausgemacht, ob die politische Kommunikationskultur in Deutschland nach wie vor gespalten und ob sie politik- oder mediengetrieben ist. Dass diese Frage ungeklärt ist, liegt u. E. auch daran, dass die vorliegenden Studien - mit Ausnahme von Kepplinger (2009a, 2009b) - wenig systematisch sind und sich auf sehr geringe Fallzahlen oder Sekundäranalysen stützen. Dadurch entsteht ein Bild der politischen Kommunikationskultur, das sich mehr auf Interpretationen verlässt als auf die systematische empirische Deskription der Einstellungen der Akteure.

\subsection{Forschungsfragen und empirische Untersuchung}

Vor diesem Hintergrund ist es das Ziel unserer Studie, die politische Kommunikationskultur in Berlin anhand der Kommunikationsorientierungen von politischen und medialen Eliten sowie von Sprechern systematisch zu analysieren. Auf der Basis einer quantitativen empirischen Erhebung sollen folgende Fragen beantwortet werden:

1. Wie unterscheiden sich Politiker, politische Sprecher und Journalisten in ihrer Einschätzung von Medien und welche Handlungsstrategien verbinden sich mit den medialen Einflussüberzeugungen?

2. Wie verstehen sie ihre eigene politische Kommunikationsrolle, wie die ihres Gegenübers?

3. Welche Konfliktpotenziale ergeben sich aus einem möglicherweise widerstrebenden Rollenselbstverständnis?

Die Träger der politischen Kommunikationskultur sind nach unserer Definition politische und journalistische Eliten, die nach den Kriterien des sog. Positionsansatzes (Bürklin 1997) in Führungsrollen der Organisationen des politischen Kommunikationssystems verankert sind. Dazu gehören Berufspolitiker der nationalen Ebene sowie Personen, die in verantwortlicher Position in der Politikvermittlung sowie der nationalen Berichterstattung darüber tätig sind. Diese Population kann in drei Gruppen unterteilt werden: Berufspolitiker, hauptamtliche Sprecher politischer Organisationen und Politikjournalisten. Zur politischen Elite zählen wir Mitglieder der Bundesregierung (z. B. Staatssekretäre bzw. Berater der Kanzlerin), Mitglieder des Bundestages mit besonderen Aufgaben (z. B. Fraktionssprecher, Bundestagspräsidium, Ausschussvorsitzende) und Funktionsträger der Parteien (z. B. Parteivorstände). Politische Sprecher sind Personen in Regierungs-, Parlaments- oder Parteiorganisationen, die die politischen Vorhaben und das öffentliche Erscheinungsbild dieser Organisationen nach außen darstellen ${ }^{4}$. Wir ha-

4 Dazu zählen wir auch die Leitungspositionen der wichtigsten Kommunikationsberatungsagenturen der politischen Parteien. 
ben uns bewusst dafür entschieden, die Gruppe der Politiker, die für sich selbst sprechen, von der Gruppe derjenigen, die für Regierung, Parteien oder einzelne Politiker sprechen, $\mathrm{zu}$ unterscheiden. Wir tun dies, weil uns bisher keine empirische Studie bekannt ist, welche die Einstellungsmuster von Politikern und ihren Sprechern systematisch vergleicht und dadurch in der Lage ist, die kognitiven Grundlagen ihres Verhältnisses zu beleuchten. Das Projekt trat mit dem Ziel einer Vollerhebung der wichtigsten Positionsinhaber im jeweiligen Sektor an. Bei der Auswahl der Befragten wurden in den jeweiligen Sektoren die relevanten Organisationen bestimmt und im zweiten Schritt innerhalb der jeweiligen Organisation die wichtigsten Ämter und Positionen. Als Ausgangsgruppe der Befragung wurden 411 politische Eliten und 117 Sprecher ausgewählt.

Auf der Seite der Medien haben wir Journalisten zur Funktionselite ihrer Zunft gezählt, wenn sie eine Spezialisierung auf den Bereich der nationalen Politik aufweisen und möglichst dauerhaft und maßgeblich an redaktionellen Entscheidungen ihrer Redaktion in Bezug auf die Themen der nationalen Politikberichterstattung und ihre Kommentierung beteiligt sind. Im Bereich des politischen Journalismus wurden 343 Positionen ausgewählt. Beim Fernsehen haben wir die Nachrichtenredaktionen, die politischen Talkshows und Magazinsendungen der öffentlich-rechtlichen Sender sowie RTL aktuell, N-TV und N24 berücksichtigt. Für das Radio wurden das Deutschlandradio sowie die Berlinkorrespondenzen der regionalen öffentlich-rechtlichen Sendeanstalten berücksichtigt. Auf der Seite der Printmedien wurden die nationalen Qualitätszeitungen (SZ, FAZ, Welt, FR, taz), Bild, sechs Regionalzeitungen ${ }^{5}$, die nationalen Wirtschaftszeitungen (FTD, Handelsblatt), die wichtigsten politischen Magazine und Wochenzeitungen sowie dpa, AP und ein Online-Nachrichtenportal (Spiegel Online) einbezogen. ${ }^{6}$ Die Interviews fanden zwischen April und Juli 2008 bzw. November 2008 und März 2009 entweder telefonisch (durch CATI), persönlich-mündlich oder mit einem Online-Fragebogen ${ }^{7}$ statt. Obwohl die Ausschöpfungsquote mit insgesamt 41 Prozent für eine Elitenbefragung durchaus zufriedenstellend ist, waren wir in der Feldphase mit großen Problemen bei der Erreichbarkeit und Teilnahmebereitschaft von Politikern konfrontiert. Daher divergieren die Ausschöpfungsquoten besonders zwischen der Gruppe der Politiker (26\%), den Sprechern (59\%) und den Journalisten (55\%) beträchtlich. Vergleiche mit den Rücklaufquoten vergleichbarer Befragungen zeigen aber, dass unsere Studie hier keine Ausnahme ${ }^{8}$ darstellt. Zwischen den Befragungsarten zeigten sich dagegen kaum Unterschiede in den Ausschöpfungsquoten ${ }^{9}$. In der Verteilung der reali-

5 Es handelt sich um die WAZ, die Rheinische Post, die Hannoversche Allgemeine, den Tagesspiegel, die Berliner Zeitung und die Leipziger Volkszeitung. Die ersten drei wurden aufgrund der Höhe der verkauften Auflage (Schütz 2007), die letzen drei aufgrund der Häufigkeit, mit der andere Medien sie zitieren (Media Tenor 2007), ausgewählt.

6 Kriterium war hier das Nachrichtenportal mit der größten Nettoreichweite gemäß www.agof.de.

7 Obwohl nicht ausgeschlossen werden kann, dass die unterschiedlichen Befragungsmodi die Vergleichbarkeit der Antworten etwas beeinträchtigen, haben wir die unterschiedlichen Optionen angeboten, um die Teilnahmebereitschaft der schwierig zu rekrutierenden Untersuchungsteilnehmer zu erhöhen.

8 Kepplinger (2009a) berichtet in einer postalischen Abgeordnetenbefragung im Jahr 2008 einen Rücklauf von 31 Prozent, Weßels (2005) erreicht eine Ausschöpfung von 34 Prozent. In einer Abgeordnetenbefragung im Jahr 1979 konnten dagegen noch 55 Prozent der Abgeordneten rekrutiert werden (Kepplinger 2009a: 68). Da wir sicherstellen wollten, dass die Zielpersonen selbst an der Studie teilnehmen, haben wir uns gegen die schriftliche Befragung entschieden und dafür eine niedrigere Ausschöpfung in Kauf genommen.

9 CATI (40\%), Online (39\%), persönlich-mündliche Befragung (35\%). 
sierten Interviews in den Befragtengruppen finden wir bei den Parlamentariern und Parteieliten eine leichte Überrepräsentation der SPD und der Linkspartei ${ }^{10}$. Bei den Journalisten zeigt die Verteilung auf die verschiedenen Tätigkeitsbereiche keine auffälligen Verzerrungen.

\section{Ergebnisse}

\subsection{Medienmacht und Aufmerksamkeitsstrategien}

Die Wahrnehmung des Einflusses der Massenmedien in Prozessen politischer Kommunikation betrifft die Frage, wie sehr sich die mediale „Logik“ als Machtfaktor der öffentlichen Meinungsbildung in das Denken von Journalisten, Politikern und ihren Sprechern „eingegraben“ hat (vgl. Altheide \& Snow 1979: 237). Um die Einflussüberzeugungen zu messen, haben wir in unserer Studie zunächst einen relativ pauschalen Indikator verwendet und nach der allgemeinen Einschätzung der Medienmacht gefragt. Möglicherweise ist aber für das konkrete Handeln und das Verhältnis von Politikern und Journalisten viel wichtiger, ob den Medien Macht über ganz spezifische Aspekte von Politik zugesprochen wird, indem sie zum Beispiel das politische Agenda-Setting oder die Personalisierung von Politik beeinflussen. Daher differenzieren wir bei den Einflussüberzeugungen auch, ob und wie stark der Medieneinfluss auf die Themensetzung in der Politik gesehen wird und ob und wie stark die Medien den Erfolg von Politikern bestimmen. Im Weiteren untersuchen wir, ob die Wahrnehmung von Machtpotenzialen der Medien mit konkreten Handlungsorientierungen und Aufmerksamkeitsstrategien der Politikdarstellung zusammenhängt.

Tabelle 1 zeigt die Verteilung derjenigen, die einen starken Medieneinfluss auf die Politik wahrnehmen. Danach unterscheiden sich Politiker, Journalisten und Sprecher signifikant voneinander. Mehr als die Hälfte der Politiker gehen von mächtigen Medien aus, während nicht einmal 20 Prozent der Journalisten von einflussreichen Medien sprechen möchten. Diese Verteilung entspricht den Befunden vergleichbarer Studien (Kepplinger 2009b, Walgrave \& Van Aelst 2006): Journalisten sind eher darum bemüht, die Wirkungsmächtigkeit der Medien zu relativieren, während Politiker das pauschale Bild „übermächtiger Medien“ gerne und mit Überzeugung aufrechterhalten. Dass Journalisten den Einfluss der Medien herunterspielen, ist insofern plausibel, als sich mit abnehmender Machtzuschreibung ihr Handlungsspielraum erhöht. Überraschend ist in unserer Studie indessen, dass auch Politiker dazu tendieren, den eigenen Einfluss zu relativieren: Nur 28 Prozent der Politiker räumen z. B. ein, dass sie Einfluss auf die Themen der öffentlichen Debatte haben, während 36 Prozent der Journalisten hier Machtpotenziale sehen.

Betrachtet man die medialen Einflussüberzeugungen in Bezug auf spezifische Aspekte wie die politische Themensetzung und den Erfolg von Politikern, so relativiert sich das Bild von den mächtigen Medien noch weiter. Nur jeweils etwa zwei Fünftel der Politiker sind der Meinung, dass die Medien die politische Agenda und den Erfolg von Politikern beeinflussen. Offenbar werden die Medien von Politikern mehr als pauschaler Machtfaktor als in ihrem konkreten Einflusspotenzial wahrgenommen. Interessant sind hier die Journalisten, die den Einfluss der Medien auch auf das politische Agenda-Setting

10 Da die Zahl der ausgewählten Positionen zwischen CDU und SPD annähernd gleich verteilt war und die Vertreter der kleinen Parteien proportional zu ihrer Verteilung in den jeweiligen Ämtern ausgewählt wurden, können wir die Verteilung auf eine höhere Verweigerungsrate der CDU-Abgeordneten und der FDP-Parteieliten zurückführen. 
Tabelle 1: Einflussüberzengungen von Medien und Politik (Prozentwerte)

\begin{tabular}{lccccl}
\hline & $\begin{array}{c}\text { Journalisten } \\
(\mathrm{n}=186)\end{array}$ & $\begin{array}{c}\text { Sprecher } \\
(\mathrm{n}=67)\end{array}$ & $\begin{array}{c}\text { Politiker } \\
(\mathrm{n}=104)\end{array}$ & $\begin{array}{c}\text { Total Cramer's V } \\
(\mathrm{n}=357)\end{array}$ \\
\hline $\begin{array}{l}\text { Medienmacht allgemein } \\
\text { Medieneinfluss auf die politische The- }\end{array}$ & 18,9 & 37,3 & 56,3 & 33,1 & $.293 * \% *$ \\
menagenda $^{2}$ & 18,3 & 29,9 & 38,5 & 26,4 & $.170 *$ \\
$\begin{array}{l}\text { Medieneinfluss auf den Erfolg von Politi- } \\
\text { kern }^{3}\end{array}$ & 39,2 & 66,7 & 41,4 & 44,9 & $.159 *$ \\
$\begin{array}{l}\text { Politikereinfluss auf die öffentliche Agen- } \\
\mathrm{da}^{4}\end{array}$ & 36,2 & 23,9 & 28,2 & 31,5 & .092 n.s. \\
\hline
\end{tabular}

$* \mathrm{p}<0,05, * * \mathrm{p}<0,01, * * \mathrm{p}<0,001$.

Zustimmung zu folgenden Items auf einer Skala $(1=$ trifft überhaupt nicht $\mathrm{zu}$ bis $5=$ trifft voll und ganz zu), ausgewiesen sind Prozentangaben der Skalenwerte „trifft eher“ und „trifft voll und ganz $\mathrm{zu}$ “.

1 „Die Macht der Medien wird überschätzt“ (Skala gedreht); ${ }^{2}$ „Die Medien und nicht die Politiker entscheiden, welche Themen in der Politik wichtig sind.“ " 3 "Ob Politiker Erfolg haben, hängt überwiegend von der Medienberichterstattung ab.“ ${ }^{4}$ „Wie erfolgreich sind Politiker im Allgemeinen darin, Einfluss auf die Themen der öffentlichen Diskussion zu nehmen?" ( 1 = überhaupt nicht erfolgreich bis $5=$ sehr erfolgreich).

herunterspielen. Gleichzeitig sind zwei Fünftel dieser Gruppe der Meinung, dass die Medien den Erfolg von Politikern maßgeblich beeinflussen. In Bezug auf die Personalisierung von Politik räumen die Journalisten also durchaus Machtpotenziale ein, während sie in Bezug auf die substanzielle politische Themensetzung einen Einfluss größtenteils von sich weisen. Die politischen Sprecher schließlich nehmen in Bezug auf das allgemeine Einflusspotenzial sowie das Themensetzungspotenzial der Medien eine Mittelposition zwischen Politikern und Journalisten ein. In ihrer Vermittlerrolle relativieren sie die pauschalen Einflussüberzeugungen von Politikern, folgen aber auch nicht dem Anschein der Machtlosigkeit der Journalisten. Wichtig ist hier, dass die Sprecher die Medienmacht in erster Linie in Bezug auf deren Personalisierungspotenzial fokussieren. Zwei Drittel der politischen Sprecher sehen einen Zusammenhang zwischen Politikererfolg und Medieneinfluss, im Vergleich zu gerade etwa 40 Prozent der Politiker und der Journalisten. Eine mögliche Erklärung ist, dass die Sprecher ihre professionelle Bedeutung in Bezug auf die Darstellung von Politikern interpretieren, während sie sich nur am Rande in den Prozess der Politikformulierung einbezogen sehen.

Die Zusammenhänge zwischen der Gruppenzugehörigkeit und der Wahrnehmung von Medienmacht sind signifikant und eindeutig in der Richtung ${ }^{11}$. Danach konstellieren sich klare Gegenpositionen zwischen Politikern und Journalisten: Journalisten bestreiten die allgemeine Medienmacht (Pearson's $-.364 * *$ ) und das Agenda-Setting-Potenzial der Medien (Pearson's $\left.-.215^{* *}\right)$, während die Politiker die Macht der Medien sowohl in Bezug auf den pauschalen Machtfaktor (Pearson's $+.325^{* *}$ ) als auch auf ihren AgendaSetting-Einfluss (Pearson's $+.155^{* *}$ ) hervorheben. In Bezug auf den Medieneinfluss auf Politikerkarrieren schließlich heben sich die Sprecher ab (Pearson's $\left.+.157^{* *}\right)$. Darüber hinaus besteht in der Gesamtpopulation der Befragten ein signifikant negativer Zusammenhang (Pearson's -.128*) zwischen der Wahrnehmung von Medienmacht und politi-

11 Die Gruppenzugehörigkeit wurde als Dummy-Variable rekodiert. Die Korrelationskoeffizienten lassen nicht nur die Stärke und Signifikanz, sondern auch die Richtung des Zusammenhangs erkennen $(* \mathrm{p}<0,05, * \mathrm{p}<0,01, \cdots * \mathrm{p}<0,001)$. 
scher Macht. D. h. je stärker der mediale Einfluss auf die politische Themenagenda eingeschätzt wird, umso schwächer wird der Einfluss der Politik auf die öffentliche Diskussion eingeschätzt. Dieser Zusammenhang ist insbesondere bei den politischen Sprechern sehr deutlich und hochsignifikant ausgeprägt (Pearson's -.297*). Auch bei den Journalisten ist die Einflusswahrnehmung dichotom programmiert, d. h. je stärker das politische Potenzial der Medien eingeschätzt wird, umso geringer wird der Politikeinfluss auf die öffentliche Diskussion wahrgenommen (Pearson's -.174*). Bemerkenswert ist, dass dieser strukturierte Zusammenhang bei den Politikern nicht nachweisbar ist. Für diese Gruppe ist die Macht der Medien oder die Macht der Politik über die Themen der öffentlichen Diskussion keine Entweder/Oder-Frage. Vielmehr stehen die beiden Aspekte für die Gruppe der Politiker sogar in einem positiven (wenn auch statistisch nicht signifikanten) Zusammenhang.

Politisch relevant werden die medialen Einflussüberzeugungen, wenn sie das Handeln von Journalisten und Politikern beeinflussen. Gibt es also einen Zusammenhang zwischen der Wahrnehmung politischer Medienmacht und den Handlungsdispositionen der Akteure in der politischen Kommunikation? Um dieser Frage nachzugehen, haben wir zunächst die Handlungsdispositionen untersucht, als Indikatoren verwenden wir die Einschätzung der Effektivität verschiedener Handlungsstrategien bei der öffentlichen Darstellung von Politik. Als klassische Strategie der Politikvermittlung operationalisieren wir die Rede im Parlament. Demgegenüber wird in der Literatur über die Medialisierung vielfach auf die Talkshowisierung von Politik (Tenscher \& Schicha 2002) als Normalzustand der politischen Kommunikation in modernen Demokratien hingewiesen. Dem Umstand, dass politische Kommunikation nicht nur auf der medialen oder parlamentarischen Vorderbühne stattfindet, trägt die Strategie des gezielten Zuspielens von Informationen an Journalisten durch Politiker Rechnung. Die komplementäre Strategie besteht in Formen der öffentlich inszenierten Dramatisierung und Konflikterzeugung, die als Aufmerksamkeitsstrategien auf der Vorderbühne gelten können, da sie die Nachrichtenfaktorenlogik antizipieren.

Die Ergebnisse in Tabelle 2 zeigen, dass die Eliten der politischen Kommunikation in Deutschland das Parlament als Forum der politischen Öffentlichkeit weitgehend abgeschrieben haben. Nicht einmal zehn Prozent der Politiker halten eine Rede im Bundestag für eine erfolgreiche Strategie der Aufmerksamkeitserzeugung von Politik. Journalisten und Sprecher glauben an diesen Ansatz sogar in noch geringerem Maße. Dies bedeutet, dass der Bundestag seine herausragende Stellung als Ort der politischen Debatte weitgehend verloren hat. Sarcinelli (2009: 240) führt dies darauf zurück, dass weite Teile des parlamentarischen Geschehens nicht mit klassischen Aufmerksamkeitskriterien in Einklang zu bringen sind. Dagegen sind sich Journalisten und Sprecher einig darin, dass die gezielte Information von Journalisten der insgesamt effektivste Ansatz ist, wenn politische Akteure öffentlich zur Kenntnis genommen werden möchten ${ }^{12}$. Politiker hingegen scheinen auf die große Reichweite und das Format von Talkshows zu setzen, denn sie glauben, dass Auftritte in diesem Medienforum die effektivste Aufmerksamkeitsstrategie ist. Der Idee, dass man vor allem die Nachrichtenlogik von Medien im Sinne von Dramatisierungsstrategien bedienen müsse, um Aufmerksamkeit zu provozieren, stimmen in allen Gruppen etwa zwei Fünftel der Befragten zu, ohne dass man hier signifikante Unterschiede zwischen den Gruppen findet. Dies kann man so interpretieren, dass über solche Strategien Konsens besteht in dem Sinne, dass sie zwar als hilfreich

12 Bei den Politikern sind es vor allem die Befragten der drei kleinen Parteien in der Opposition, die auf das Einflusspotenzial des Zuspielens von Informationen an die Medien setzen. 
gesehen werden, dass sie aber nicht den durchschlagenden Erfolg in Bezug auf öffentliche Aufmerksamkeit versprechen.

\section{Tabelle 2: Strategien öffentlicher Aufmerksamkeitserzeugung für politische Themen (Prozentwerte)}

\begin{tabular}{lrrrrr}
\hline $\begin{array}{l}\text { Strategien der öffentlichen Aufmerksam- Journalisten } \\
\text { keitserzeugung für politische Themen }{ }^{1}\end{array}$ & $\begin{array}{r}\text { Sprecher Politiker } \\
(\mathrm{n}=187)\end{array}$ & $\begin{array}{r}\text { Total } \\
(\mathrm{n}=67)\end{array}$ & $\begin{array}{r}\text { Cramer's } \\
(\mathrm{n}=358)\end{array}$ & $\mathrm{V}$ \\
\hline Journalisten Informationen zuspielen & 72,2 & 88,1 & 65,4 & 73,2 & $.172^{* * *}$ \\
Talkshow-Auftritte & 49,5 & 49,2 & 72,1 & 56,1 & $.161^{*}$ \\
Dramatisierte Darstellung & 35,6 & 47,8 & 42,7 & 40,0 & n.s. \\
Rede im Bundestag & 6,4 & 3,0 & 9,7 & 6,8 & n.s. \\
\hline
\end{tabular}

$* \mathrm{p}<0,05, * \mathrm{p}<0,01, \cdots * \mathrm{p}<0,001$.

${ }^{1}$ Zustimmung zu folgenden Items (eher wirkungsvoll, sehr wirkungsvoll): Politiker versuchen auf unterschiedlichsten Wegen, öffentliche Aufmerksamkeit für ihre Anliegen zu wecken. Ich lese Ihnen jetzt einige Möglichkeiten vor. Bitte sagen Sie mir jeweils, aus Ihrer Sicht, für wie wirkungsvoll Sie diese halten $(1=$ überhaupt nicht wirkungsvoll bis $5=$ sehr wirkungsvoll).

Betrachtet man die einzelnen Berufsgruppen separat, zeigt sich einerseits, dass der Glaube an die Macht der Medien bei Politikern in der Tat dazu führt, das große Forum der reichweitenstarken Medienformate als Bühne zu suchen. In der Tat finden wir signifikante Zusammenhänge zwischen der Zugehörigkeit zur Gruppe der Politiker und der Wahrnehmung, dass Talkshows die effektivsten Kommunikationsmodi für Politik sind (Pearson's +.199*). Darüber hinaus zeigt eine Faktorenanalyse ${ }^{13}$, in die die Variablen der Einflussüberzeugung (Tabelle 1) und der Aufmerksamkeitsstrategien (Tabelle 2) eingingen, dass die Machtwahrnehmung von Medien auf der gleichen Dimension mit der Wahrnehmung von Talkshows als Modi effektiver Politikdarstellung liegt. Die Politiker scheinen sich darauf eingestellt zu haben, dass sich Medienmacht in diesem Format manifestiert. Diese Einschätzung wird definitiv nicht von den Journalisten geteilt, wie ein signifikant negativer Zusammenhang zwischen der Gruppenzugehörigkeit zu den Medien und der Einschätzung der Effektivität von Talkshows (Pearson’s -.152*\%) zeigt. Danach betrachten die Medienvertreter die Talkshows deutlich zurückhaltender, wenn es um die effektive öffentliche Darstellung von Politik geht.

Die Journalisten, deren Interesse die exklusive Information ist, und die Sprecher, die ihre Spins und Interpretationen möglichst ungefiltert in den Medien sehen wollen, halten das direkte Zuspielen von Informationen für die effektivste Öffentlichkeitsstrategie. Dieser Befund zeigt, dass die Wahl von probaten Strategien der Herstellung von Aufmerksamkeit die eigenen Zielsetzungen jeder Gruppe markiert. Interessant ist hier, dass auch die Sprecher stärker auf Aspekte des politisch-strategischen Nahverhältnisses von Medien und Politik abheben (Pearson's $+.186^{* *}$ ), also eher einer politischen Logik der Hinterbühne das Wort reden, während Politiker eher die mediale Vorderbühnenstrategie bevorzugen. Dies entspricht Befunden, welche für politische Sprecher einen Rollenwandel weg von der nach außen gerichteten Verlautbarungskommunikation hin zur Stabilisierung des politisch-medialen Beziehungsgeflechts auf den Hinterbühnen politischer Öffentlichkeit konstatieren (Tenscher 2003: 219). Die strategische Schnittstelle

13 Extraktion durch Hauptkomponentenanalyse, rotiert (Varimax mit Kaiser-Normalisierung); 3-Faktoren-Lösung, berücksichtigt sind Faktorladungen > .5; erklärte Gesamtvarianz 52,5 Prozent. 
zwischen Medien und Politik jenseits der öffentlichen Arena, welche politische Sprecher ihrem Selbstverständnis nach besetzen, halten sie folgerichtig auch für besonders effektiv, wenn es um die Generierung von Aufmerksamkeit für politische Themen geht.

Unsere Interpretation wird auch durch die Faktorenanalyse von Einflussüberzeugungen und Öffentlichkeitsstrategien unterstützt. Die Hinterbühnenstrategien des Zuspielens von Informationen an Journalisten und die Dramatisierung von Informationen liegen auf der gleichen Dimension wie die Einstellung einer starken Einflussüberzeugung der Politik auf die öffentliche Agenda. Die Orientierung, dass politischer Einfluss auf die Öffentlichkeit eher von einer aus ihrer Sicht „effektiven“ Informationsversorgung von Journalisten abhängt, erscheint in diesem Sinne als die Geschäftsgrundlage zwischen politischen Sprechern und Medien. Offenbar ist die politische Kommunikationskultur in Berlin geprägt von einer Arbeitsteilung zwischen Politikern und ihren Sprechern: Während die Sprecher das Nahverhältnis und die direkte Informationsversorgung von Journalisten suchen, gehen die Politiker selbst den Weg der großen Öffentlichkeit und suchen die Vorderbühne von Talkformaten in der Wahrnehmung, auf die starke Medienmacht zu reagieren.

\subsection{Selbstverständnis und Rollenbilder}

In Bezug auf die professionellen Selbst- und Fremdeinschätzungen untersuchen wir, wie stark sich die Wahrnehmung der eigenen politischen Kommunikationsrolle von Politikern und Journalisten mit der Fremdwahrnehmung dieser Rolle durch das jeweilige Gegenüber deckt bzw. wie groß die Diskrepanzen sind. Dabei fragen wir nicht nach dem normativen Verständnis, d. h. welche Erwartungen die jeweils andere Gruppe erfüllen soll, sondern welche Rollen für die jeweils andere Gruppe tatsächlich wichtig sind. Bei der Wahl der empirischen Indikatoren für journalistische Rollen greifen wir auf die zentralen Studien der Kommunikatorforschung zurück (Donsbach \& Patterson 2003, Weaver 1998, Weaver \& Wilhoit 1991, Weischenberg et al. 2006). Da für Politiker keine vergleichbaren Indikatoren über ihre Kommunikationsrollen vorliegen, beziehen wir uns in der Operationalisierung auf die Kommunikationsziele bei Blumler/Gurevitch ${ }^{14}$ (1995: 240ff.). Bei den politischen Sprechern wurde jeweils die Fremdwahrnehmung der journalistischen und der politischen Rolle erfragt.

Tabelle 3 zeigt die Rollen der Journalisten und die Einschätzung der korrespondierenden Gruppen. Danach betonen die Journalisten für sich selbst in fast gleich starkem Ausmaß die Rolle des Informationsvermittlers wie auch die der Kontrolle der Politik. Die Zustimmung zu diesen Zielen ist etwas stärker als zur Wichtigkeit einer ausgewogenen Berichterstattung. Diesen „politischen“ Rollen nachgeordnet sind zunächst die „publizistischen“ Normen der Publikumsorientierung sowie der Anspruch der Aktualität. Bemerkenswert ist schließlich, dass die eigene politische Meinungsäußerung in der Selbstwahrnehmung der Journalisten den geringsten Stellenwert hat. Die von den Journalisten proklamierten Motivlagen werden von den Politikern und Sprechern in weitaus geringerem Maße als Ziele des politischen Journalismus wahrgenommen, wie die Gruppenunterschiede zwischen der Selbstzuschreibung und der Fremdzuschreibung zeigen. Die positiven Vorzeichen verdeutlichen hier, dass die Journalisten die jeweiligen Rollen für deutlich prägender halten als die Politiker. Insbesondere bezweifeln die Politiker,

14 Da die Items somit bisher nicht empirisch validiert wurden, haben wir diesen Teil der Untersuchung durch eine offene Nachfrage ergänzt. Wir wollten vermeiden, dass wir substanzielle Rollenbilder politischer Akteure in der politischen Kommunikation durch die geschlossene Frageform ausschließen. 
dass für die Journalisten das Ziel der Informationsvermittlung im Vordergrund steht. Vielmehr unterstellen sie den Journalisten die Absicht, ihre eigene politische Meinung verbreiten zu wollen. Politiker unterstellen Journalisten deutlich stärker politische Motive, als diese das selbst für sich in Anspruch nehmen.

Tabelle 3: Journalistische Rollen (Mittelwerte, Saldi)

\begin{tabular}{lccccr}
\hline Journalistische Rollen & \multicolumn{3}{c}{ Gruppe (Mittelwerte) } & & $\begin{array}{r}\text { Saldo Eigen- vs. Fremd- } \\
\text { bild }\end{array}$ \\
\hline & $\begin{array}{c}\text { Journalisten } \\
(\mathrm{n}=185)\end{array}$ & $\begin{array}{c}\text { Sprecher } \\
(\mathrm{n}=67)\end{array}$ & $\begin{array}{r}\text { Politiker } \\
(\mathrm{n}=98)\end{array}$ & Eta & Journalisten - Politiker \\
\hline Kontrolle der Politik $^{1}$ & 4,6 & 3,0 & 3,0 & $.705^{* * *}$ & $+1,6$ \\
Informationsvermittlung $^{2}$ & 4,5 & 2,7 & 2,7 & $.756^{* * *}$ & $+1,8$ \\
Ausgewogenheit $^{3}$ & 4,3 & 2,7 & 2,9 & $.698^{* * *}$ & $+1,4$ \\
Publikumsorientierung $^{4}$ & 4,1 & 4,0 & 3,8 & $.121^{\text {n.s. }}$ & $+0,3$ \\
Aktualität $^{5}$ & 3,9 & 3,7 & 3,7 & $.104^{\text {n.s. }}$ & $+0,2$ \\
Eigene politische Ansich- $^{\text {ten }}{ }^{6}$ & 2,7 & 3,1 & 3,2 & $.206^{* *}$ & $-0,5$ \\
\hline
\end{tabular}

F-Test $(* \mathrm{p}<0,05, * \mathrm{p}<0,01, * * \mathrm{p}<0,001)$.

Wenn Sie über Politik berichten, wie wichtig ist es Ihnen (Journalisten) bzw. Wenn Journalisten über Politik berichten, ist es ihnen wichtig (Politiker/Sprecher), ${ }^{1}$...Aussagen von Politikern nachzuprüfen und die Politiker insgesamt zu kontrollieren? ${ }^{2}$...den Bürgern alle Informationen zur Verfügung zu stellen, die sie benötigen, um sich begründete politische Urteile bilden zu können? ${ }^{3}$ ...verschiedene Sichtweisen ausgewogen zu berücksichtigen? ${ }^{4}$... Nachrichten zu produzieren, die für ein möglichst breites Publikum von Interesse sind? ${ }^{5}$... dem Publikum möglichst schnell Informationen zu vermitteln? ${ }^{6}$... dem Publikum eigene Ansichten zu politischen Entwicklungen zu präsentieren? Antwortmöglichkeiten jeweils von $1=$ für mich überhaupt nicht wichtig bis $5=$ für mich sehr wichtig bzw. von $1=$ trifft überhaupt nicht zu bis $5=$ trifft voll und ganz zu.

\section{Tabelle 4: Dimensionen der Selbst und Fremdwabrnehmung journalistischer Rollen}

\begin{tabular}{lll}
\hline & Journalisten & Politiker/Sprecher \\
\hline Faktor 1 & Publikumsorientierung & Publikumsorientierung \\
& Schnelligkeit/Aktualität & Schnelligkeit/Aktualität \\
Faktor 2 & Informationsvermittlung & Informationsvermittlung \\
& Kritik und Kontrolle & Ausgewogenheit \\
Faktor 3 & Eigene Ansichten & Eigene Ansichten \\
& & Kritik und Kontrolle \\
\hline
\end{tabular}

Extraktion durch Hauptkomponentenanalyse, rotiert (Varimax mit Kaiser-Normalisierung); dargestellt sind alle Faktorladungen > .5; erklärte Gesamtvarianz 69,7 Prozent (Journalisten) sowie 71,6 Prozent (Politiker/Sprecher).

Um zu prüfen, wie die Selbst- und Fremdwahrnehmungen journalistischer Rollen zusammenhängen, haben wir die Einstellungsitems für jede Gruppe anhand einer Faktorenanalyse dimensioniert. Tabelle 4 zeigt, dass die Selbst- und Fremdwahrnehmungen in Bezug auf die publizistischen Kategorien der Publikumsorientierung und der Aktualität in ihrer Struktur übereinstimmen. Dieser Faktor markiert den Konsens zwischen Journalisten und politischen Akteuren. Unterschiede ergeben sich jedoch in Bezug auf 
die politischen Rollen: Hier wird deutlich, dass die Journalisten die Funktion der Informationsvermittlung mit der Kritik- und Kontrollfunktion verbinden und diesen Aspekt von der eigenen Meinungsäußerung streng trennen. Die Politiker hingegen verbinden die Informationsvermittlung mit Ausgewogenheit und Neutralität und assoziieren die Kritik- und Kontrollfunktion mit dem Motiv der Meinungsäußerung. Für sie geht die Watchdog-Rolle der Journalisten eindeutig mit dem Ausdruck eigener politischer Ansichten einher.

In Bezug auf die Kommunikationsrollen von Politikern haben wir auf Basis der theoretischen Vorüberlegungen von Blumler/Gurevitch (1995: 15) vier Indikatoren für Motive identifiziert, deren Wichtigkeit eingestuft werden sollte: die Rolle eines Informationslieferanten der Bürger, die des politischen Entscheiders, die des Parteipolitikers, sowie schließlich individuelle Karrieremotive. Tabelle 5 zeigt, dass sich die Politiker in ihrer Kommunikation als Parteisoldaten und fast genauso stark als Informationslieferanten der Bürger ${ }^{15}$ verstehen. An dritter Stelle werden individuelle Karrieremotive als Ziele der Kommunikation eingeräumt und schließlich die direkte Einflussnahme auf politische Entscheidungen über die Medien. Fast spiegelbildlich ist die Wahrnehmung der Journalisten: Sie unterstellen den Politikern in erster Linie individuelle Karriereabsichten als Motiv für ihr Kommunikationsverhalten und die Absicht, politische Entscheidungen zu beeinflussen. Mit wenig Abstand zu diesen Funktionen nehmen sie die Politiker als Parteisoldaten wahr, am wenigsten unterstellen sie das Motiv der politischen Aufklärung. Wiewohl die Gruppenunterschiede in Bezug auf die Rollenbilder von Politikern weniger ausgeprägt sind als in Bezug auf die Journalisten, finden wir doch eindeutige Muster der Gewichtung von Motivlagen auf beiden Seiten. Die Journalisten unterstellen den Politikern stärker politische und vor allem individuelle Motive, in den offenen Nachfragen insbesondere persönliche Eitelkeit.

\section{Tabelle 5: Kommunikationsrollen von Politikern (Mittelwerte, Saldi)}

\begin{tabular}{lccccr}
\hline $\begin{array}{l}\text { Kommunikationsrollen von } \\
\text { Politikern }\end{array}$ & \multicolumn{3}{c}{ Gruppe (Mittelwerte) } & & $\begin{array}{r}\begin{array}{r}\text { Saldo Eigen- vs. } \\
\text { Fremdbild }\end{array} \\
\hline\end{array}$ \\
\hline & $\begin{array}{c}\text { Politiker } \\
(\mathrm{n}=100)\end{array}$ & $\begin{array}{r}\text { Journalisten } \\
(\mathrm{n}=184)\end{array}$ & $\begin{array}{c}\text { Sprecher } \\
(\mathrm{n}=65)\end{array}$ & Eta & $\begin{array}{r}\text { Politiker - Journalis- } \\
\text { ten }\end{array}$ \\
\hline Parteipolitiker $^{1}$ & 4,2 & 4,0 & 4,1 & $.113 \mathrm{n} . \mathrm{s}$. & $+0,2$ \\
Informationslieferant $^{2}$ & 4,1 & 3,4 & 3,6 & $.337^{* * * *}$ & $+0,7$ \\
Individuelle Karriere $^{3}$ & 3,7 & 4,3 & 4,1 & $.314^{* * * *}$ & $-0,6$ \\
Politischer Entscheider $^{4}$ & 3,6 & 4,0 & 3,9 & $.236^{* * * *}$ & $-0,4$ \\
\hline
\end{tabular}

F-Test $(* \mathrm{p}<0,05, * * \mathrm{p}<0,01, * * \mathrm{p}<0,001)$

Wie wichtig ist es Ihnen, in den Medien aufzutreten (Politiker) bzw. Politiker treten vor allem in den Medien auf (Journalisten/Sprecher), um ${ }^{1}$...für die Ansichten ihrer Partei zu werben. ${ }^{2}$...die Bevölkerung über ihre politischen Vorhaben zu informieren. ${ }^{3}$...um sich selbst bekannt zu machen. ${ }^{4}$...um über die Medien politische Entscheidungen zu beeinflussen. Antwortmöglichkeiten jeweils von $1=$ überhaupt nicht wichtig bis $5=$ sehr wichtig bzw. von $1=$ trifft überhaupt nicht zu bis $5=$ trifft voll und ganz zu.

15 Auch in den offenen Nachfragen betonen die befragten Politiker vorrangig ihre Bedeutung als Informationsvermittler, die sie als Aufklärung der Bürger, Stärkung der Demokratie oder die Förderung öffentlicher Meinungsbildung betrachten. 
Fasst man die Befunde zusammen, so sind auf beiden Seiten die Diskrepanzen in Selbstund Fremdwahrnehmung da am größten, wo beide Gruppen ihre eigene Rolle idealisieren. Politische Sprecher nehmen eine Mittelposition zwischen Politikern und Journalisten ein. In ihrer Wahrnehmung der Rollen beider Seiten sind sie jedoch dem Verständnis der Politiker näher als dem der Journalisten. Sie teilen im Wesentlichen die Fremdwahrnehmung der Politiker auf die Journalisten. Im Abgleich von Selbst- und Fremdbild zeigt sich die Nähe zu den Politikern erneut. Während im Durchschnitt die Sprecher in ihrer Fremdwahrnehmung nur 0,3 Punkte vom Selbstbild der Politiker abweichen, beträgt die durchschnittliche Differenz zum Selbstbild der Journalisten immerhin 0,9 Punkte. Danach können Sprecher die Motivlagen der politischen Akteure besser einschätzen als die der Journalisten.

\subsection{Konfliktwahrnehmungen}

Unsere bisherigen Befunde verweisen darauf, dass die Akteure der politischen Kommunikation in Berlin nur zum Teil in ihren Einschätzungen des Gegenübers übereinstimmen. Insbesondere aber scheint das Verhältnis auch von Unterstellungen geprägt zu sein, bei denen sich angesichts unterschiedlicher Interessen Konfliktpotenziale entwickeln können. Fragt man die politischen und medialen Eliten nach der Einschätzung ihres Verhältnisses, so finden wir auch hier signifikante Unterschiede.

\section{Tabelle 6: Wabrnehmung des Verbältnisses von Journalisten und Politikern (Prozentwerte)}

\begin{tabular}{lrrrr}
\hline Konfliktwahrnehmung $^{1}$ & $\begin{array}{r}\text { Journalisten } \\
(\mathrm{n}=183)\end{array}$ & $\begin{array}{r}\text { Sprecher } \\
(\mathrm{n}=67)\end{array}$ & $\begin{array}{r}\text { Politiker } \\
(\mathrm{n}=100)\end{array}$ & $\begin{array}{r}\text { Gesamt } \\
(\mathrm{n}=349)\end{array}$ \\
\hline Verhältnis ist eher/sehr harmonisch & 16,1 & 65,7 & 41,9 & 33,1 \\
Teils-teils & 62,8 & 29,9 & 46,9 & 51,9 \\
Verhältnis ist eher/sehr konflikthaltig & 21,1 & 4,5 & 11,3 & 15,1 \\
\hline
\end{tabular}

Cramer's V $.337 * * *$ ( $<$ <.001).

${ }^{1}$ Ganz allgemein gesprochen: Würden Sie Ihren Umgang mit Politikern (bzw. Journalisten) als eher konflikthaltig oder als eher harmonisch bezeichnen? Antwortmöglichkeit von 1 = sehr konflikthaltig bis $5=$ sehr harmonisch. Zusammengefasst wurden die Kategorien 1 und 2, sowie 4 und 5.

Wie Tabelle 6 zeigt, finden gut doppelt so viele Politiker (42\%) wie Journalisten (16\%), dass ihr gegenseitiges Verhältnis eher harmonisch sei. Umgekehrt gilt, dass fast doppelt so viele Journalisten (21\%) als Politiker (11\%) den Umgang als eher konflikthaltig bezeichnen. Für die politischen Sprecher scheint hingegen das Verhältnis zu den Medien ausgesprochen freundlich, da zwei Drittel dieser Gruppe von ausschließlicher Harmonie sprechen. Diese Einstellungsmuster verweisen darauf, dass vor allem die Politiker und ihre Sprecher auf der Vorderbühne Konflikte eher herunterspielen, während Journalisten einen etwas skeptischeren Blick auf die Interaktion haben. Vor dem Hintergrund professionsspezifischer Normen der Objektivität und Unabhängigkeit wahren Journalisten in ihrer Selbstverortung also eine eher kritische Distanz zur politischen Zunft, während sich die Nähe zu Journalisten für Politiker und politische Sprecher in ihrer eigenen Wahrnehmung weit unproblematischer darstellt. Sowohl für Politiker als auch ihre Sprecher lässt sich argumentieren, dass vielmehr ein allzu antagonistisches Verhältnis zu den Medien einen Widerspruch zum eigenen Selbstverständnis darstellt - für die 
Politiker zu ihrem Anspruch, für ihre Vorhaben öffentlich zu werben, und für die Sprecher zu ihrem professionellen Verständnis als Scharnier zwischen Medien und Politik.

Um dieses Bild noch weiter zu differenzieren, haben wir den Einfluss weiterer Variablen auf die Wahrnehmung der Konflikthaltigkeit des Verhältnisses in einem linearen Regressionsmodell (vgl. Tabelle 7) geprüft. Neben der Akteursgruppe wurden Variablen des persönlichen Kontaktverhältnisses, des wahrgenommenen Einflusses politischer Überzeugungen auf das Verhältnis, die politische Links-Rechts-Selbsteinstufung der Akteure sowie Alter und Geschlecht als Kontrollvariablen einbezogen. Die Akteursgruppe erweist sich weiterhin als der wesentliche Bestimmungsfaktor der Konfliktwahrnehmung: Sprecher nehmen das Verhältnis signifikant harmonischer, Journalisten konflikthaltiger wahr. Darüber hinaus ist jedoch ebenso relevant, ob das Verhältnis zwischen Journalisten und Politikern als von politischen Überzeugungen bestimmt wahrgenommen wird ${ }^{16}$ : Je prägender politische Überzeugungen für das eigene Verhältnis zu Politikern bzw. Journalisten erscheinen, desto konfliktreicher wird das Verhältnis zu ihnen wahrgenommen. Dabei ist der Anteil derer, die einen solchen Einfluss politischideologischer Überzeugungen eher oder sehr stark wahrnehmen, mit knapp 12 Prozent aller Befragten bereits sehr klein. Die abnehmende Wirkkraft politisch-weltanschaulicher Orientierungen und parteipolitischer Verbindungen im Verhältnis medialer und politischer Akteure ist in dieser Lesart also ein Faktor, der ein harmonisches Verhältnis zwischen beiden Seiten begünstigt. Im Gegensatz zur Wahrnehmung der Akteure, dass politische Überzeugungen für das Medien-Politik-Verhältnis relevant sind, bleibt die tatsächliche politisch-ideologische Orientierung jedoch ohne Erklärungskraft: die Selbst-Einstufung auf der Links-Rechts-Skala hat keine signifikante Auswirkung auf die Wahrnehmung des Konfliktgrades der Beziehung zwischen medialen und politischen Akteuren ${ }^{17}$.

\section{Tabelle 7: Lineare Regressionsanalyse (standardisierte Beta-Werte)}

Wahrgenommene Konflikthaltigkeit des Verhältnisses ${ }^{1)}$

\begin{tabular}{|c|c|}
\hline \multirow{2}{*}{\multicolumn{2}{|c|}{ Gruppenzugehörigkeit (Politiker als Basiswert) }} \\
\hline & \\
\hline Journalisten (Dummy) & $.217 * \%$ \\
\hline Sprecher (Dummy) & $-.135^{*}$ \\
\hline Persönliches Kontaktverbältnis & $.139 * \%$ \\
\hline Häufigkeit beruflichen persönlichen Kontakts & .010 \\
\hline Anzahl Freunde unter Politikern bzw. Journalisten & $-.210 * * *$ \\
\hline Einfluss politischer Überzeugungen auf das Verhältnis & $.151 * \%$ \\
\hline $\begin{array}{l}\text { Politische Links-Rechts-Selbsteinstufung }(1 \text { = linker End- } \\
\text { punkt, } 7 \text { = rechter Endpunkt })\end{array}$ & -.047 \\
\hline Alter & -.049 \\
\hline Geschlecht ( 0 = männlich, 1 = weiblich $)$ & -.022 \\
\hline Korrigiertes R-Quadrat & .190 \\
\hline \multicolumn{2}{|l|}{$\begin{array}{l}* \mathrm{p}<.05, * \mathrm{p}<.01, * * \% \mathrm{p}<.001 \\
\text { 1) Skala gedreht: } 1=\text { sehr harmonisch bis } 5=\text { sehr konfliktreich. }\end{array}$} \\
\hline \multicolumn{2}{|c|}{$\begin{array}{l}\text { Gefragt wurde: „Wie stark wird Ihre professionelle Beziehung zu Politikern (Journalisten) } \\
\text { durch deren politische Überzeugungen beeinflusst?" ( } 1=\text { sehr schwach bis } 5=\text { sehr stark). }\end{array}$} \\
\hline \multicolumn{2}{|c|}{$\begin{array}{l}17 \text { Ebenso hat die Parteizugehörigkeit der politischen Akteure keine Auswirkung auf die Wahr- } \\
\text { nehmung des Konfliktverhältnisses. }\end{array}$} \\
\hline
\end{tabular}


In Bezug auf die Frage, wie sehr ein bestehendes persönliches Kontaktverhältnis zu Journalisten bzw. Politikern die Konfliktwahrnehmung beeinflusst, ergibt sich ein gemischtes Bild. Wie zu erwarten, führt ein freundschaftlich-persönliches Verhältnis, gemessen durch die Anzahl der Freunde innerhalb der jeweils anderen Akteursgruppe, zu einer signifikant harmonischeren Wahrnehmung des Verhältnisses zwischen politischen und medialen Akteuren. Allein die Häufigkeit eines beruflichen persönlichen Kontakts mit Journalisten bzw. Politikern wirkt sich dagegen noch nicht positiv auf die Harmonieeinschätzung des Verhältnisses aus. Umgekehrt bedeutet dies jedoch auch, dass eine persönliche Zusammenarbeit zwischen Journalisten und Politikern auch dann denkbar und möglich ist, wenn ihr Verhältnis zumindest latent von Konflikten geprägt ist.

Fragt man schließlich nach den Ursachen möglicher Spannungen, so zeigt sich, dass Politiker, die zunächst angeben, in Harmonie mit Journalisten zu leben, bei detaillierter Nachfrage eine ganze Reihe von Konflikten nennen ${ }^{18}$. Dazu gehört die Wahrnehmung, dass es häufiger Probleme bei der Weitergabe bzw. der Berichterstattung von Informationen gebe und Absprachen mit Journalisten nicht eingehalten würden. Insbesondere monieren Politiker und noch häufiger politische Sprecher, dass es Fehler in der Berichterstattung über von ihnen weitergegebenen Informationen gebe. Journalisten dagegen betrachten die Interaktion mit Politikern wesentlich nüchterner. In ihrer Wahrnehmung ist das Verhältnis zwischen beiden Seiten vorrangig von grundsätzlich divergierenden Interessen geprägt, welche naturgemäß Spannungen mit sich bringen. Die erhebliche Diskrepanz zwischen vorgeblicher allgemeiner Harmonie auf der Vorderbühne und dem Vorkommen einer Reihe von Konflikten insbesondere in der Wahrnehmung von Politikern zeigt, wie brüchig das Verhältnis zwischen Journalisten und Politikern tatsächlich ist. Insbesondere wenn man die Ergebnisse zur Wahrnehmung des Konfliktverhältnisses mit den Diskrepanzen in der Wahrnehmung der jeweiligen beruflichen Rolle in Verbindung setzt, wird deutlich, dass die Nähe zwischen Journalisten und Politikern in Berlin nur vordergründig ist.

\section{Diskussion und Schlussfolgerungen}

In unserer Studie haben wir die Kommunikationsorientierungen von Politik- und Medieneliten sowie politischen Sprechern in Berlin untersucht und den Versuch gemacht, die kognitive Geschäftsgrundlage der Kommunikation politischer Botschaften zu beschreiben. Zentral war die Frage, wie sehr die Orientierung an Medienlogiken tatsächlich ein durchgängiges Merkmal der politischen Kommunikationsbeziehung zwischen Politikern und Journalisten ist. Tatsächlich liefern die wenigen vorliegenden Studien, die Einstellungsmuster politischer Kommunikationsakteure in den Fokus stellen, hierzu widersprüchliche Ergebnisse und nähren somit den Zweifel an einer durchgreifenden und konsistenten Medienorientierung politischer Kommunikation in Deutschland. Wir vermuteten daher, dass aller Mediatisierungsprozesse zum Trotz sowohl in Bezug auf die Wahrnehmung des medialen Einflusses auf die Politik als auch im individuellen Selbstverständnis der Akteure weiterhin erhebliche Diskrepanzen in den Einstellungen medialer und politischer Akteure bestehen. Die Konsequenz wäre eine politische Kommunikationskultur, die von der Spannung zwischen politischer Logik und publizistischen Motiven geprägt ist.

Wir können auf Basis unserer empirischen Beschreibung tatsächlich Diskrepanzen in den Orientierungen von Politikern und Journalisten konstatieren, wenn auch in etwas 
anderer Prägung als eingangs vermutet. So manifestieren sich die Unterschiede zwischen den Gruppen gerade nicht in der Weise, dass bei den Journalisten lediglich die publizistischen Imperative gelten und auf der Seite von Politikern nur die politischen Kalküle eine Rolle spielen. Vielmehr werden die Wahrnehmungen über medienorientierte Aufmerksamkeitsstrategien und Rollenkonzepte auf beiden Seiten geteilt - die „kognitive Geschäftsgrundlage“ politischer Kommunikation scheint hier intakt. Gespalten ist die Kommunikationskultur jedoch an jenen Stellen, an denen (macht)politische Logiken ins Spiel kommen. So ist der Blick auf das Machtgefüge politischer Kommunikation systematisch verzerrt - beide Seiten sehen sich mit einer vermeintlichen Übermacht der anderen Seite konfrontiert, die Wahrnehmung eines Harmonieverhältnisses schwindet mit dem Einfluss politischer Überzeugungen in der Interaktion. Das Verständnis für die Rolle des Gegenübers endet dort, wo es dem eigenen demokratischen Idealbild der jeweiligen Gruppe am nächsten kommt. Die Betrachtung der Konfliktdimension zeigt jedoch, dass die verschiedenen Gruppen sehr unterschiedlich mit auftretenden Diskrepanzen und Spannungen umgehen. Während Politiker versuchen, das Bild vermeintlicher Harmonie zumindest auf der Vorderbühne aufrechtzuerhalten, ziehen sich Journalisten auf eine Position zurück, nach welcher die Einstellungsunterschiede zwischen beiden Seiten Ausdruck des professionellen Interessensgegensatzes sind.

Fragt man nach den Konsequenzen der so beschriebenen Berliner Kommunikationskultur für den Zustand politischer Öffentlichkeit, so ist die vermeintliche Konkordanz auf der medialen bzw. mediatisierten Vorderbühne politischer Kommunikation mit Skepsis zu betrachten. Allzu leicht vermag sie darüber hinwegzutäuschen, dass das Verhältnis beider Seiten weiterhin von unterschwelligen Diskrepanzen geprägt ist. Diese lassen den Umgang von Politikern, ihren Sprechern und Journalisten brüchiger erscheinen, als in der Diskussion übergreifender Mediatisierungs- und Professionalisierungstendenzen oft angenommen wird. Schließlich haben wir in unserer Studie mit den politischen Sprechern eine weitere Gruppe ins Spiel gebracht, deren Rolle weit über einen „verlängerten Arm“ der Politik hinausgeht. Politische Sprecher heben sich in ihren Einschätzungen deutlich von politischen Entscheidungsträgern ab, sind aber auch nicht auf eine reine Mittlerposition zwischen der politischen und medialen Seite beschränkt. Sie zeigen an einigen Stellen vielmehr ganz eigene, spezifische Wahrnehmungen auf politische Kommunikationsprozesse, deren Konsequenz bisher kaum systematisch untersucht ist.

\section{Literatur}

Altheide, D. L. \& Snow, R. P. (1979). Media Logic. Beverly Hills: Sage.

Baugut, P., \& Grundler, M.-T. (2009). Politische (Nicht-)Öffentlichkeit in der Mediendemokratie. Eine Analyse der Beziehungen zwischen Politikern und Journalisten in Berlin. Baden-Baden: Nomos Verlag.

Blumler, J., \& Gurevitch, M. (1981). Politicians and the Press. An Essay on Role Relationships. In D. D. Nimmo \& K. R. Sanders (Eds.), Handbook of Political Communication. Beverly Hills: Sage, $467-493$.

Blumler, J. G., \& Gurevitch, M. (1995). The Crisis of Public Communication. London/New York: Routledge.

Bruns, T. (2007). Republik der Wichtigtuer: Ein Bericht aus Berlin. Freiburg: Herder.

Bürklin, W. (1997). Die Potsdamer Elitestudie von 1995: Problemstellungen und wissenschaftliches Programm. In W. Bürklin \& H. Rebenstorf (Hrsg.), Eliten in Deutschland. Rekrutierung und Integration. Opladen: Leske + Budrich, 11-34. 
Donsbach, W., Jarren, O., Kepplinger, H. M., \& Pfetsch, B. (1993). Beziehungsspiele - Medien und Politik in der öffentlichen Diskussion. Fallstudien und Analysen. Gütersloh: Verlag Bertelsmann Stiftung.

Donsbach, W., \& Patterson, T. (2003). Journalisten in der politischen Kommunikation: Professionelle Orientierungen von Nachrichtenredakteuren im internationalen Vergleich. In F. Esser \& B. Pfetsch (Eds.), Politische Kommunikation im internationalen Vergleich. Grundlagen, Anwendungen, Perspektiven. Wiesbaden: Westdeutscher Verlag, 281-304.

Esmark, A., \& Mayerhöffer, E. (2010). Shaping the Public Agenda-Professional Political Communication between Media Logic and Political Logic. Paper presented at the Annual Conference of the International Communication Association, Singapore, 22.-26.06.2010.

Hachmeister, L. (2007). Nervöse Zone. Politik und Journalismus in der Berliner Republik. München: Deutsche Verlags-Anstalt.

Hallin, D., \& Mancini, P. (2004). Comparing Media Systems. Three Models of Media and Politics. Cambridge: Cambridge University Press.

Hanitzsch, T. (2007). Journalismuskultur: Zur Dimensionierung eines zentralen Konstrukts der kulturvergleichenden Journalismusforschung. $M E K, 3,372-389$.

Harmgarth, F. (1997). Wirtschaft und Soziales in der politischen Kommunikation. Eine Studie zur Interaktion von Abgeordneten und Journalisten. Studien zur Kommunikationswissenschaft. Opladen: Westdeutscher Verlag.

Hoffmann, J. (2003). Inszenierung und Interpenetration: Das Zusammenspiel von Eliten aus Politik und Journalismus. Wiesbaden: Westdeutscher Verlag.

Jarren, O., Donges, P., \& Weßler, H. (1996). Medien und politischer Prozess. Eine Einleitung. In O. Jarren, H. Schatz \& H. Weßler (Eds.), Medien und politischer Prozess. Politische Öffentlichkeit und massenmediale Politikvermittlung im Wandel. Opladen: Westdeutscher Verlag, 9-37.

Kepplinger, H. M. (2009a). Politikvermittlung. Wiesbaden: VS.

Kepplinger, H. M. (2009b). Rivalität um Macht und Moral: Bundestagsabgeordnete und Hauptstadtjournalisten. In H. Kaspar, H. Schoen, S. Schumann \& J. R. Winkler (Eds.), Politik - Wissenschaft-Medien. Festschrift für Jürgen W. Falter zum 65. Geburtstag. Wiesbaden VS, 307-322.

Lesmeister, C. (2008). Informelle politische Kommunikationskultur. Hinter den Kulissen politischmedialer Kommunikation. Wiesbaden: VS Verlag.

Maurer, P. (2011). Explaining Perceived Media Influence in Politics. An Analysis of the Interplay of Context and Attitudes in four European Democracies. Erscheint in Publizistik 56 (1).

Maurer, P., \& Mayerhöffer, E. (2009). Themenmanagement von politischen Sprechern und Kommunikationsberatern unter den Bedingungen der Bonner und der Berliner Republik. Ein Zeitvergleich 1994-2008. Zeitschrift für Politikberatung, 2, 447-466.

Mayerhöffer, E. (2009). The Perception of Public Opinion Polls by Journalists and Politicians in Eight European Countries. Paper presented at the 5th ECPR General Conference, Potsdam, 10.-12.09.2009.

Mazzoleni, G. (1987). Media Logic and Party Logic in Campaign Coverage: The Italian General Election 1983. European Journal of Communication, 2, 81-103.

Media Tenor (2007). Forschungsbericht Nr. 157 - I/2007.

Pfetsch, B. (2003a). Politische Kommunikationskultur - ein theoretisches Konzept zur vergleichenden Analyse politischer Kommunikationssysteme. In F. Esser \& B. Pfetsch (Eds.), Politische Kommunikation im internationalen Vergleich. Grundlagen, Anwendungen, Perspektiven. Wiesbaden: Westdeutscher Verlag, 393-418.

Pfetsch, B. (2003b). Politische Kommunikationskultur. Politische Sprecher und Journalisten in der Bundesrepublik und den USA im Vergleich. Wiesbaden: Westdeutscher Verlag.

Pfetsch, B. (2008). Political Communication Culture. In W. Donsbach (Ed.), International Encyclopedia of Communications. Oxford: Blackwell, 3683-3686.

Pfetsch, B. \& Marcinkowski, F. (2009). Problemlagen der "Mediendemokratie" - Theorien und Befunde zur Medialisierung von Politik. In F. Marcinkowski \& B. Pfetsch (Eds.), Politik in der Mediendemokratie. Wiesbaden: VS Verlag, 11-33.

Pfetsch, B., Maurer, P. \& Mayerhöffer, E. (2009). A Hedge Between Keeps Friendship Green. Concurrence and Conflict between Politicians and Journalists in Western Democracies. Paper pre- 
sented at the 59th Annual Conference of the International Communication Association Chicago, 21.-24.05.2009.

Plasser, F., \& Lengauer, G. (2010). Politik vor Redaktionsschluss: Kommunikationsorientierungen von Macht- und Medieneliten in Österreich. In F. Plasser (Ed.), Politik in der Medienarena: Praxis politischer Kommunikation in Österreich. Wien: Fakultas, 53-100.

Rinke, E. M., Schlachter, M., Agel, F., Freund, C., Götz, T., Täuber, U., et al. (2006). Netzwerk Berlin. Informelle Interpenetration von Politik und Journalismus. München: Martin Meidenbauer Verlagsbuchhandlung.

Rolke, L., \& Wolff, V. W. (Eds.) (2003). Die Meinungsmacher in der Mediengesellschaft. Deutschlands Kommunikationseliten aus der Innensicht. Wiesbaden: VS.

Sarcinelli, U. (2009). Politische Kommunikation in Deutschland: Zur Politikvermittlung im demokratischen System. Wiesbaden: VS Verlag, 2. Auflage.

Schütz, W. J. (2007). Deutsche Tagespresse 2006. Trotz Anzeigen- und Auflagenverlusten kaum Zeitungskrise spürbar. Media Perspektiven (11), 560-588.

Schulz, W. (2008). Politische Kommunikation. Theoretische Ansätze und Ergebnisse empirischer Forschung (2. Aufl.). Wiesaden: VS Verlag.

Schwab Cammarano, S., Donges, P., \& Jarren, O. (2010). Politische Kommunikationskultur im Wandel. Studies in Communication Sciences, 10 (1), 7-21.

Tenscher, J. (2003). Professionalisierung der Politikvermittlung? Politikvermittlungsexperten im Spannungsfeld von Politik und Massenmedien. Wiesbaden: Westdeutscher Verlag.

Tenscher, J., \& Schicha, C. (Eds.) (2002). Talk auf allen Kanälen: Angebote, Akteure und Nutzer von Fernsehgesprächssendungen. Wiesbaden: Westdeutscher Verlag.

Walgrave, S., \& Van Aelst, P. (2006). The Contingency of the Mass Media's Political Agenda Setting Power: Toward a Preliminary Theory. Journal of Communication, 56, 88-109.

Weaver, D. (1998). The Global Journalist: News People Around the World. Cresskill: Hampton Press.

Weaver, D. H., \& Wilhoit, G. C. (1991). The American Journalist. A Portrait of U.S. News People and Their Work (2nd. Ed.). Bloomington: Indiana University Press.

Weischenberg, S., Malik, M., \& Scholl, A. (2006). Die Souffleure der Mediengesellschaft. Report über die Journalisten in Deutschland. Konstanz: UVK.

Weßels, B. (2005). Abgeordnetenbefragung 2003. Kurzfassung und Dokumentation der Ergebnisse. http://www.wzb.eu/zkd/dsl/pdf/ber-fin1-all1.pdf [29.12.2010].

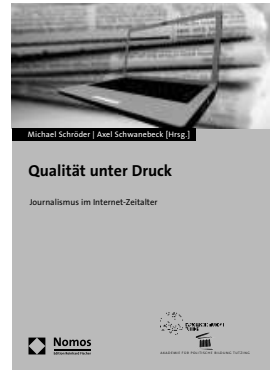

Bitte bestellen Sie im Buchhandel oder versandkostenfrei unter $\bullet$ www.nomos-shop.de

\section{Qualität unter Druck}

Journalismus im Internet-Zeitalter

Herausgegeben von Michael Schröder und

Axel Schwanebeck

2011, 183 S., brosch., 19,80€

ISBN 978-3-8329-6055-1 